\section{RASPBERRY PI BASED WEATHER STATION}

\author{
A.C. GHEORGHE, M.S. CHIRAN \\ Department of Electronics, Telecommunications and Energy Engineering, Valahia University of Targoviste \\ E-mail: gheorghe_andrei89@yahoo.com
}

\begin{abstract}
The paper proposes the development of a weather station made up from a Raspberry Pi 3 and the Sense Hat shield. The shield used in this application has sensors for temperature, humidity and pressure. The weather station can connect to the internet wirelessly or with a standard UTP (Unshielded twisted pair) connection, this connection gives us the ability to take part in a scientific community for the sole purpose to monitor the weather all around the world. The program for the weather station is made in Python, the program language is easy to used and very versatile.
\end{abstract}

Keywords: Raspberry Pi, shield Sense Hat, Wireless, Python

\section{INTRODUCTION}

The paper proposes the development of a weather station made up from a Raspberry Pi 3 and the Sense Hat shield. The shield used in this application has sensors for temperature, humidity and pressure. The weather station can connect to the internet wirelessly or with a standard UTP (Unshielded Twisted Pair) connection, this connection gives us the ability to take part in a scientific community for the sole purpose to monitor the weather all around the world. The program for the weather station is made in Python, the program language is easy to used and very versatile [1]. Components that are used in this application are:

- Raspberry Pi 3 that is used to run the program in Python and connect wirelessly or wired to the scientific community via Internet;

- Sense Hat Shield that is used to obtain data like: humidity, temperature and atmospheric pressure, the shield can be used for other applications.

\subsection{Raspberry Pi 3}

The Raspberry Pi 3 is a development board used in this application, we can see the mechanical schematic in Figure 1 and the overall look of the board in Figure 2, the development board has GPIO pins that are used to connect with the Sense Hat for collecting data and providing power to the shield as we can see in Figure 3 [2].

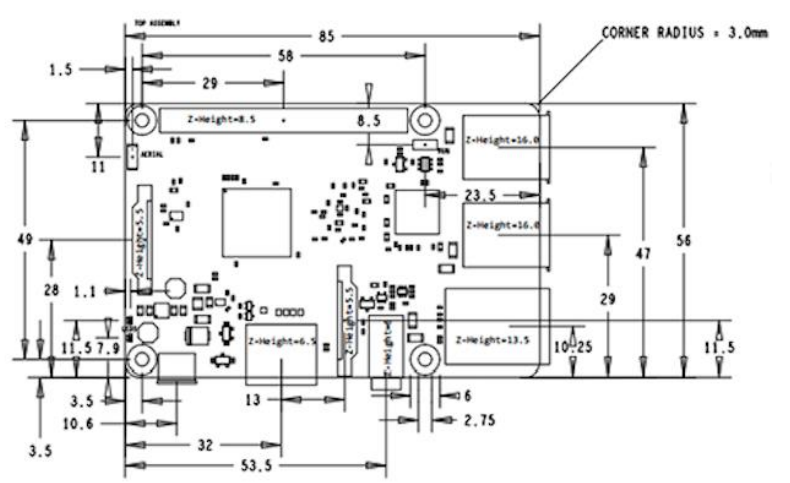

Figure 1. Mechanical schematic

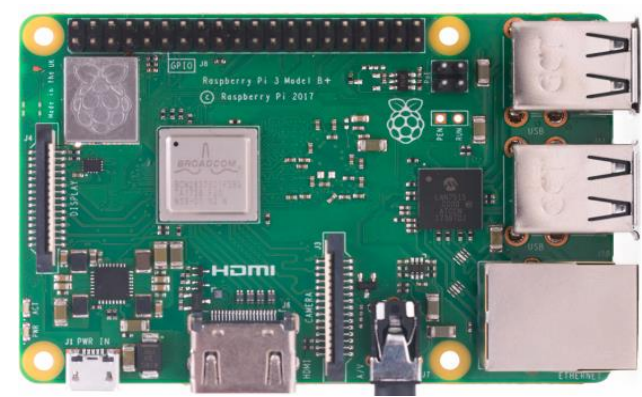

Figure 2. Raspberry Pi 3 overall look

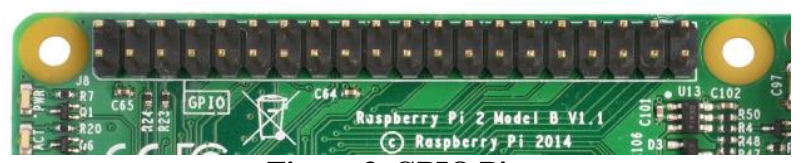

Figure 3. GPIO Pins

The GPIO (general-purpose input/output) pins are a powerful feature on the Raspberry Pi 3. They offer a variety of analog and digital connections as we can see in Figure 4.

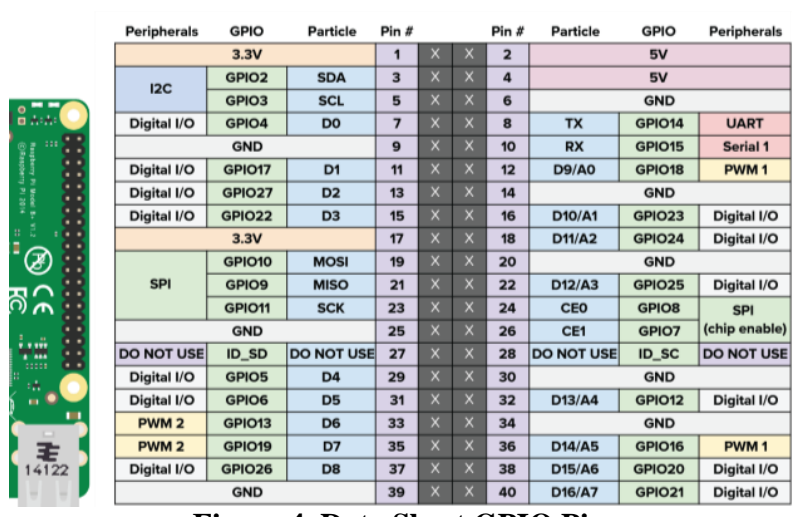

Figure 4. Data Sheet GPIO Pins 
The Raspberry PI 3 development board has a $1.2 \mathrm{GHz}$ quad-core ARM CPU, 1 GB DDR 2 RAM and other specifications as we can see in Figure 5.

\begin{tabular}{|c|c|}
\hline Processor. & $\begin{array}{l}\text { Broadcom BCM2837B0, Cortex-A53 } \\
\text { 64-bit SoC @ 1.4GHz }\end{array}$ \\
\hline Memory. & 1GB LPDDR2 SDRAM \\
\hline Connectivity. & $\begin{array}{l}2.4 \mathrm{GHz} \text { and } 5 \mathrm{GHz} \text { IEEE } 802.11 \mathrm{~b} / \mathrm{g} / \mathrm{n} / \mathrm{ac} \text { wireless } \\
\text { LAN, Bluetooth } 4.2, \mathrm{BLE} \\
\text { Gigabit Ethernet over USB } 2.0 \text { (maximum throughput } \\
300 \mathrm{Mbps} \text { ) } \\
\text { - } 4 \times \text { USB } 2.0 \text { ports }\end{array}$ \\
\hline Access: & Extended 40-pin GPIO header \\
\hline Video \& sound: & $\begin{array}{l}1 \times \text { full size HDMI } \\
\text { MIPI DSI display port } \\
\text { MIPI CSI camera port } \\
\text { " } 4 \text { pole stereo output and composite video port }\end{array}$ \\
\hline Multimedia: & $\begin{array}{l}\text { H.264, MPEG-4 decode }(1080 \text { p } 30) ; ~ H .264 \text { encode } \\
\text { (1080p30); OpenGL ES 1.1, } 2.0 \text { graphics }\end{array}$ \\
\hline SD card support: & $\begin{array}{l}\text { Micro SD format for loading operating system and } \\
\text { data storage }\end{array}$ \\
\hline Input power. & $\begin{aligned} & 5 \mathrm{~V} / 2.5 \mathrm{~A} \text { DC via micro USB connector } \\
& 5 \mathrm{~V} D C \text { via GPIO header } \\
& \text { - } \text { Power over Ethernet (PoE)-enabled (requires } \\
& \text { separate PoE HAT) }\end{aligned}$ \\
\hline Environment: & Operating temperature, $0-50^{\circ} \mathrm{C}$ \\
\hline
\end{tabular}

Figure 5. Raspberry Pi 3 specifications

\subsection{Sense Hat Shield}

The Sense HAT shield as we can see in Figure 6 is attached to the top of Raspberry Pi through the 40 GPIO pins (which provide data and the power interface) to create the weather station. Sense HAT has multiple sensors on the integrated circuit that can be used for many different types of experiments, applications, and even games. Having the following technical specifications as we can see in Figure 7 [3].

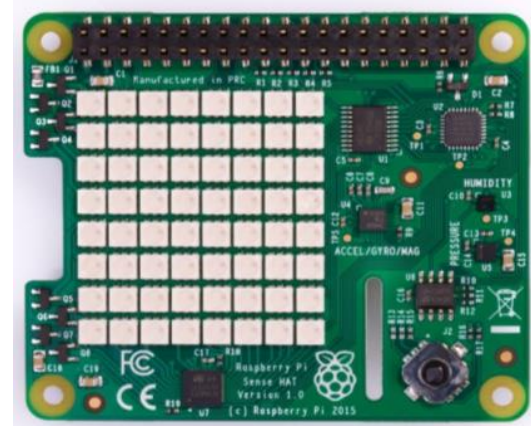

Figure 6. Sense Hat Shield

\section{TECHNICAL DETAILS}

- Gyroscope

- Angular rate sensor (dps): 245/500/2000

- Accelerometer

- Linear acceleration sensor $(\mathrm{g}):$ 2/4/8/16

- Magnetometer

- Magnetic sensor (gauss): $4 / 8 / 12 / 16$

- Barometer: $260-1260 \mathrm{hPa}$ absolute range (accuracy depends on the temperature and pressure, ${ }^{\sim} 0.1 \mathrm{hPa}$ under normal conditions)

- Temperature sensor: Accurate to ${ }^{\circ} 2^{\circ} \mathrm{C}$ in the $0-65^{\circ} \mathrm{C}$ range

- Relative humidity sensor: Accurate to $\sim 4.5 \%$ in the $20-80 \% \mathrm{rH}$ range, accurate to $\sim 0.5^{\circ} \mathrm{C}$ in

$15-40^{\circ} \mathrm{C}$ range

- $8 \times 8$ LED matrix display

- Small 5 button joystick

Figure 7. Sense Hat Data Sheet

\section{WEATHER STATION PROGRAM}

In Figure 8 we can see the initialization procedure for the upload configuration, after having succeeded it passes to the next stage and gets the values from the Sense Hat. The ID provided by the personal weather network is taken from a secondary configuration file and if the program gets all that it needs, proceeds to starts the initialization of the Sense Hat.

The program starts with an initial temperature taken from the shield, were the temperature, humidity and pressure values can be observed. The time to transmit the information to the weather site is set at 1 minute interval, the user can set the interval up to 60 minutes and the program works in a infinite loop [4].

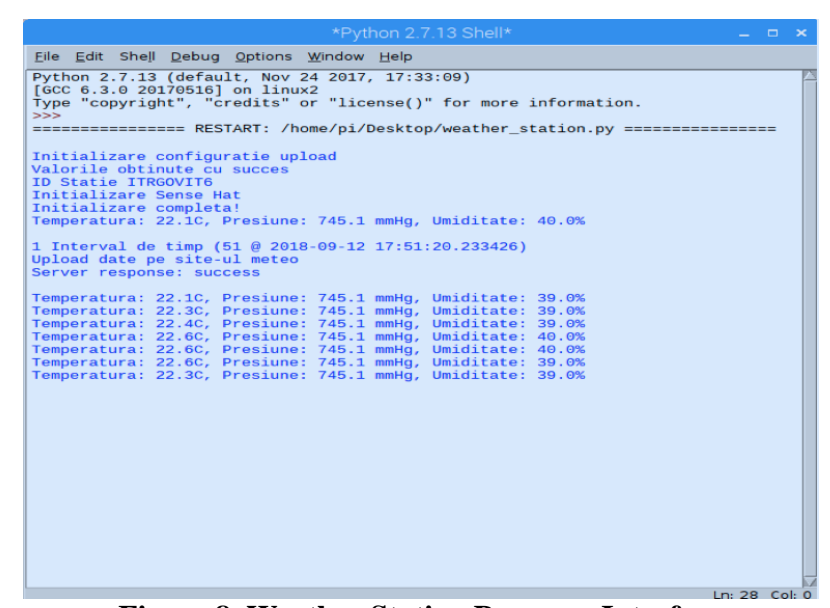

Figure 8. Weather Station Program Interface

In the Figure 9 we can see that the station is online, functional and transmits information to the scientific community to observe the weather. To use the station in the scientific community we had to use the "https://www.wunderground.com/" site where we could create a personal account. ID and Key were provided by this site and it was easy to implement with our application.

\section{Personal Weather Station Network}

Overview Buying Guide Register with WU

Personal Weather Stations

\section{Your Registered Stations}

If you are moving your PWS more than a few blocks from its current location, you need to sign up for a new station ID.

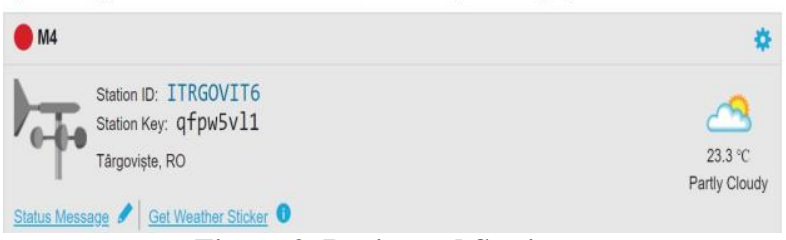

Figure 9. Registered Station 


\section{EXPERIMENTS}

\subsection{Experiment No.1}

In Figure 10 we can see the covered weather station with a microfiber cloth and we can notice increasing temperatures, variations in pressure and humidity (Figure 11), as a reference I took the information from a room thermometer. In this experiment, we observe that the temperature is quite accurate to the room thermometer used.

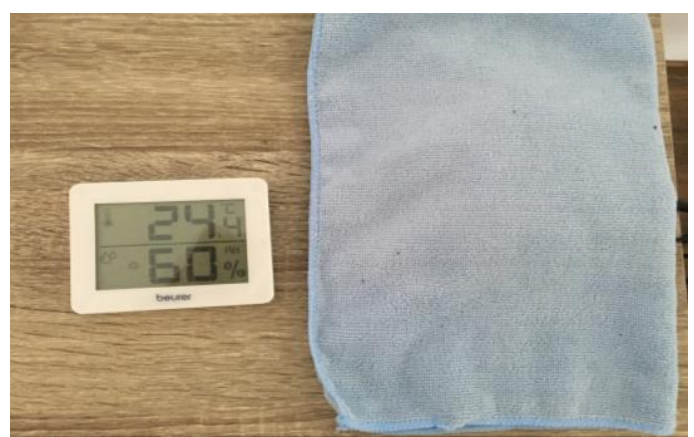

Figure 10. Covered Weather Station

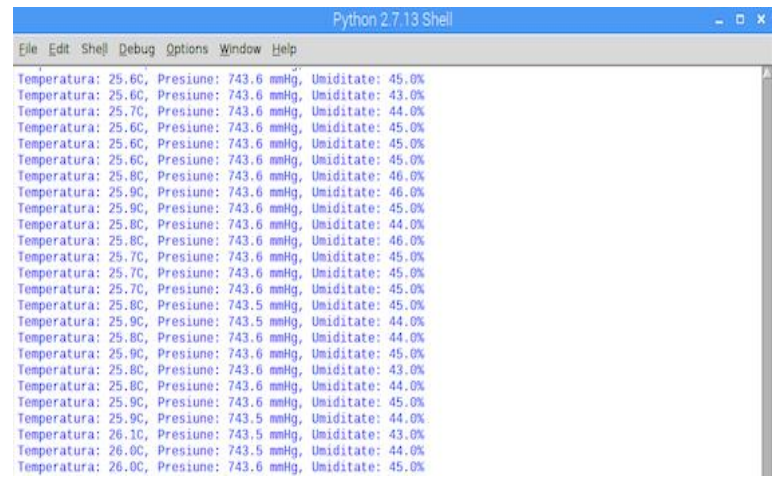

Figure 11. Readings from the experiment No.1

\subsection{Experiment No.2}

In Figure 12 we can see a box that is covering the weather station and we can observe a slightly rise in temperature and small variation in atmospheric pressure and humidity (Figure 13).

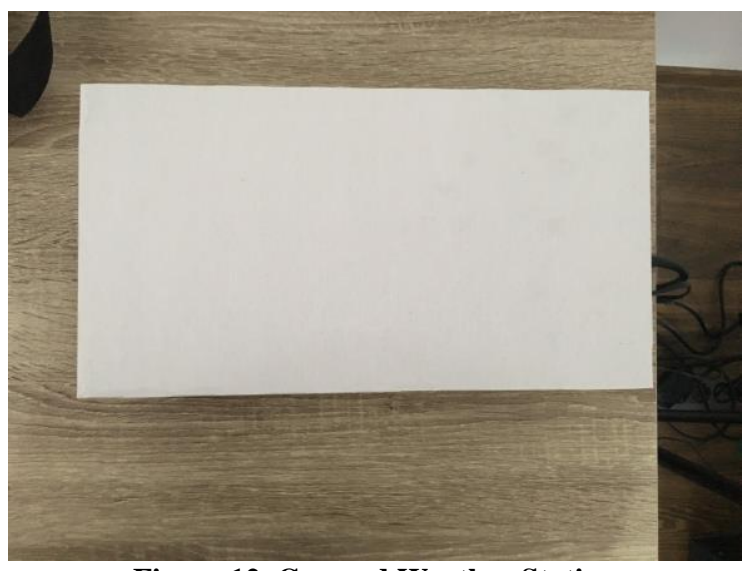

Figure 12. Covered Weather Station

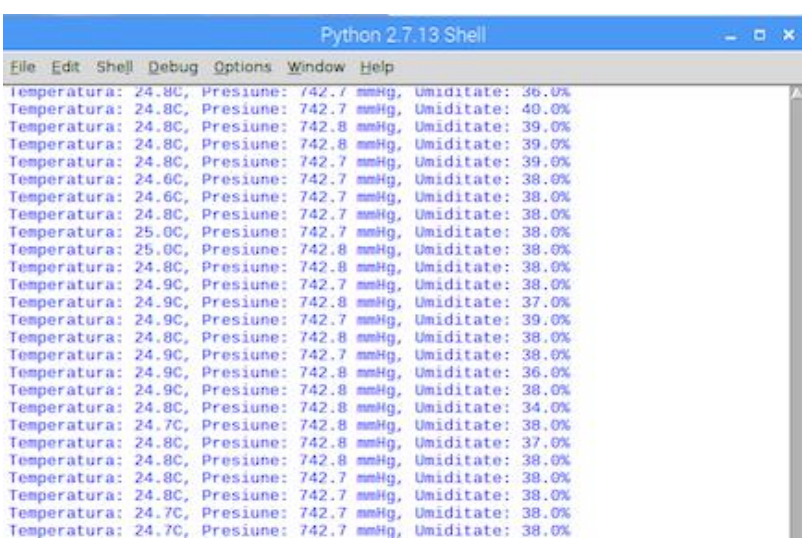

Figure 13. Readings from the experiment No.2

\subsection{Experiment No.3}

In Figure 14 we started the A.C and let it run for about 30-40 minutes, we noticed a decrease in the temperature equivalent to that displayed by the room thermometers taken as a reference, the difference was 1 degree Celsius (error of $4.54 \%$ ) and the temperature and humidity of $12 \%$ (30\% error) (Figure 15).

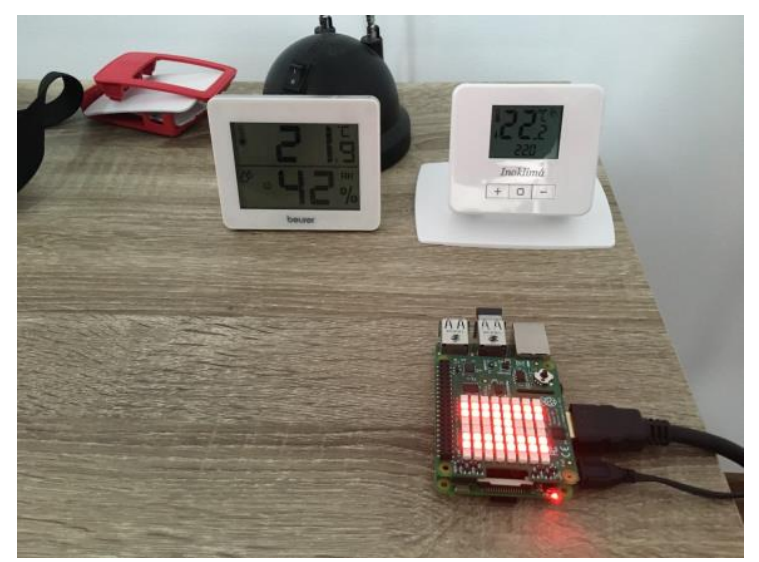

Figure 15. Weather Station and two room thermometers

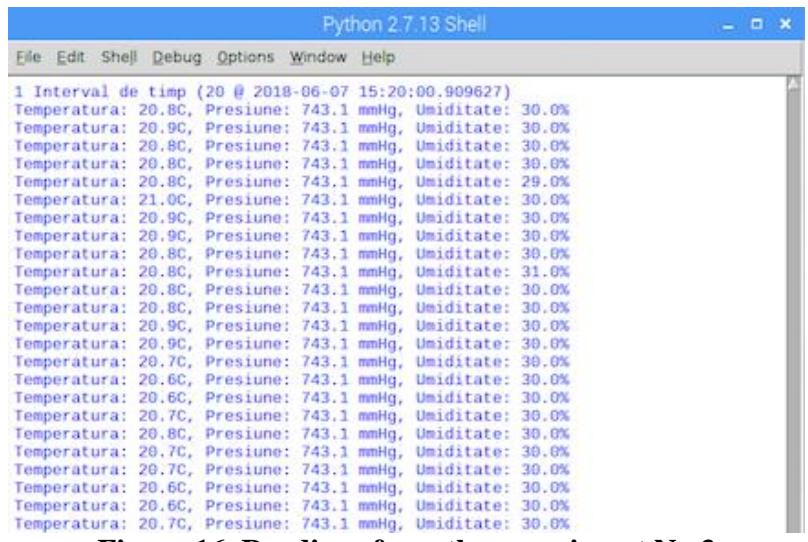

Figure 16. Readings from the experiment No.3

\subsection{Experiment No.4}

In Figure 17 we used a hair dryer to heat the weather station and the room thermometer for a few seconds and found that the measurements in Figure 18 are very close, 
but the precision reference is still given by a digital camera thermometer containing the temperature sensor and humidity in a closed space.

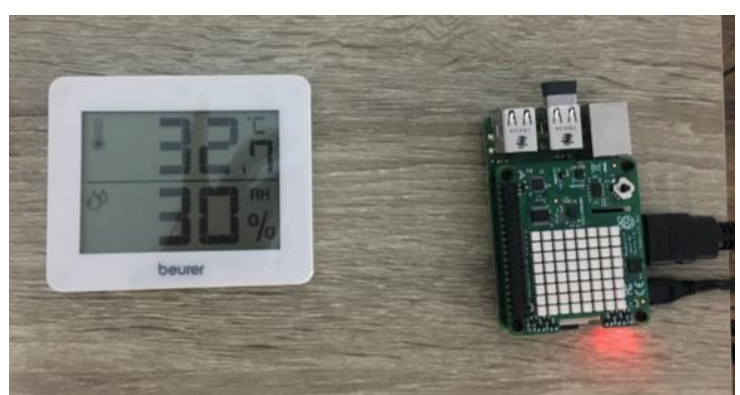

Figure 17. Weather Station and room thermometer

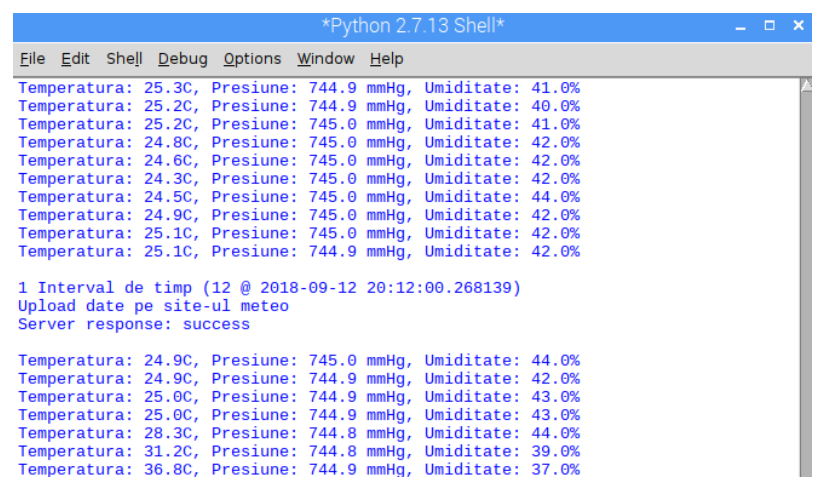

Figure 18. Readings from experiment No.4

\section{CONCLUSIONS}

The application is useful to keep track of weather, and the weather station can connect to the internet by adding it to a scientific community made up of several weather stations built from Raspberry Pi+ Sense Hat to monitor weather around the world. From the experiments we noticed small differences between the device and the thermometers used as a reference point, such as: at a temperature of $4.5 \%$ error and quite high humidity differences with a $30 \%$ error in some experiments, but it is in generally $3-5 \%$ error rate.

\section{REFERENCES}

[1] E. Matthes, Python Crash Course: A Hands-On, Project-Based Introduction to Programming, No Starch Press, 2015.

[2] K. Kearney, Creative Projects with Raspberry Pi, Hatchette Co., 2017.

[3] Al Swigart, Automate the Boring Stuff With Python: Practical Programming for Total Beginners, No Strach press, 2017.

[4] B. Lubanovic, Introducing Python: Modern Computing in Simple Packages, O'Reilly Media Inc., 2015. 